Bull. Austral. Math. Soc.

VoL. 47 (1993) [341-346]

\title{
FURTHER RESULTS ON THE DEFICIENCIES OF ALGEBROID FUNCTIONS
}

\section{LIANZHONG YANG}

Let $f(z)$ be an $n$-valued algebroid function of finite lower order $\mu$. In this paper, we give some further results on the deficiencies of $f(z)$. Particularly if $0 \leqslant \mu \leqslant 1 / 2$, the corresponding result is best possible.

\section{INTRODUCTION}

Let $f(z)$ be an $n$-valued algebroid function of finite lower order $\mu$, defined by an irreducible equation

$$
A_{0} f^{n}+A_{1} f^{n-1}+\cdots+A_{n-1} f+A_{n}=0
$$

where $A_{0}, A_{1}, \ldots, A_{n}$ are entire functions without common zeros, and we assume that the reader is familiar with the fundamental concepts of Nevanlinna's theory and adopt, with their usual meaning, classical symbols such as (see $[1,4,5]$ )

$$
N(r, f), T(r, f), \delta(a, f), \sigma(a, f), \ldots .
$$

In a previous paper, Yang [6] established an inequality (Spread Relation):

$$
\sigma(a, f) \geqslant \min \left\{2 \pi, \frac{4}{\pi} \arcsin \sqrt{\frac{\delta(a, f)}{2}}\right\}
$$

and prove the following theorem.

THEOREM A. Let $f(z)$ be an $n$-valued algebroid function of lower order $\mu(0 \leqslant \mu<\infty)$. Then on summing over all the deficient values $a$ of $f(z)$, we have

$$
\sum_{a} \sqrt{\delta(a, f)} \leqslant n(\sqrt{2} \mu \pi+2 \mu+1) .
$$

It is well known that if $f(z)$ is an entire function of lower order $\mu(0 \leqslant \mu \leqslant 1 / 2)$, then $f(z)$ has no finite deficient values. This and Theorem A suggest the following problems for a $n$-valued algebroid function $f(z)$ :

(a) What is the best possible upper bound of $\sum \delta(a, f)$ when $f(z)$ is of lower order $\mu(0 \leqslant \mu \leqslant 1 / 2)$ ?

(b) What is the best possible upper bound of $\sum \sqrt{\delta(a, f)}$ where the $\sum$ is the summation over all deficient values of $f(z)$ ?

Received 29 April 1992

This research was supported by a grant from NSF of Peoples Republic China

Copyright Clearance Centre, Inc. Serial-fee code: 0004-9729/93 \$A2.00+0.00. 
In the present paper, we shall prove

THEOREM 1. Let $f(z)$ be a $n$-valued algebroid function of finite lower order $\mu$. Then on summing over all the deficient values $a$ of $f(z)$, we have

$$
\sum_{a} \sqrt{\delta(a, f)} \leqslant \begin{cases}\frac{\pi \mu n}{\sqrt{2}} & \left(\frac{\sqrt{2}}{\pi}<\mu<+\infty\right) \\ n & \left(0 \leqslant \mu \leqslant \frac{\sqrt{2}}{\pi}\right) .\end{cases}
$$

ThEOREM 2. Let $f(z)$ be an $n$-valued algebroid function of finite lower order $\mu$, then

$$
\sum_{a} \delta(a, f) \leqslant n, \quad\left(0 \leqslant \mu \leqslant \frac{1}{2}\right) .
$$

\section{Two Lemmas}

LеммA 1. Let $f(z)$ be an $n$-valued algebroid function of lower order $\mu(0<\mu<\infty)$, then

$$
\sum_{a} \min \left\{2 \pi, \frac{4}{\mu} \arcsin \sqrt{\frac{\delta(a, f)}{2}}\right\} \leqslant 2 n \pi,
$$

where the $\sum_{a}$ is the summation over all the deficient values $a$ of $f(z)$.

Proof: By the spread relation (2), Lemma 1 is a rewritten form of a theorem due to Yang [6, Theorem 2.1].

In order to state a lemma of Edrei [2], we assume that

$$
x=\varphi(s) \quad(0 \leqslant s \leqslant 1)
$$

is a real continuous function satisfying the following conditions

(1) $\varphi(0)=0, \varphi(1)=1$.

(2) $\varphi^{\prime}(s)$ and $\varphi^{\prime \prime}(s)$ exist for $0<s<1$, and they are strictly positive and continuous in the interval $(0,1)$.

Denote by $\psi(x)$ the inverse function:

$$
s=\psi(x)=\varphi^{-1}(x), \quad(0 \leqslant x \leqslant 1) .
$$

LEMma 2. Let the quantities $x_{j}(j=1,2, \ldots, k ; n+1 \leqslant k \leqslant \infty)$ be subject to the constraints

$$
0 \leqslant x_{j} \leqslant 1 \quad(1 \leqslant j \leqslant k), \quad \sum_{j=1}^{k} \psi\left(x_{j}\right) \leqslant H<\infty,
$$

then

$$
\sum_{j=1}^{k} x_{j} \leqslant[H]+\varphi(H-[H])
$$


Equality is possible in (4) if and only if $k<\infty$ and

(1) exactly $[H]$ of $x$ are equal to 1 ;

(2) one $x$ is $\varphi(H-[H])$;

(3) all other $x$, if they exist, are equal to 0.

\section{Proof of Theorem 1}

We consider the following three cases.

CASE (A). $\mu=0$. By a result of $\mathrm{Gu}[3], f(z)$ has at most $n$ deficient values and Theorem 1 follows in this case.

CASE (B). $\mu>1 / 2$. We denote by $E=\{a: \delta(a, f)>0\}$ the set of all the deficient values of $f(z)$. By Lemma 1, we have

$$
\sum_{a \in E} \min \left\{2 \pi, \frac{4}{\mu} \arcsin \sqrt{\frac{\delta(a, f)}{2}}\right\} \leqslant 2 n \pi .
$$

It is obvious that our assumption $\mu>1 / 2$ implies

$$
\frac{4}{\mu} \arcsin \sqrt{\frac{\delta(a, f)}{2}}<2 \pi
$$

so it follows from (5) that

$$
\sum_{a \in E} \frac{4}{\mu} \arcsin \sqrt{\frac{\delta(a, f)}{2}} \leqslant 2 n \pi .
$$

Hence, from an elementary triangular inequality, we can deduce that

$$
\sum_{a \in E} \sqrt{\delta(a, f)} \leqslant \sqrt{2} \sum_{a \in E} \arcsin \sqrt{\frac{\delta(a, f)}{2}} \leqslant \frac{\mu \pi}{\sqrt{2}} n .
$$

This proves Theorem 1 in Case (b).

CASE (c). $0<\mu \leqslant 1 / 2$. Let the set $E$ be defined as in Case (b). By (5) it is easily seen that there are at most $n$ elements $a$ of $E$ such that

$$
\frac{4}{\mu} \arcsin \sqrt{\frac{\delta(a, f)}{2}} \geqslant 2 \pi \text {. }
$$

Denoting by $E_{0}$ the set of values $a$ such that (6) holds, we deduce from (5) and (6) that

$$
\sum_{a \in E-E_{0}} \frac{4}{\mu} \arcsin \sqrt{\frac{\delta(a, f)}{2}} \leqslant 2(n-k) \pi,
$$


where $k$ is the number of element in $E_{0}$.

Similar to case (b) of the proof of Theorem 1, we have

(7)

$$
\begin{aligned}
\sum_{a \in E} \sqrt{\delta(a, f)} & =\sum_{a \in E-E_{0}} \sqrt{\delta(a, f)}+\sum_{a \in E_{0}} \sqrt{\delta(a, f)} \\
& \leqslant \sqrt{2} \sum_{a \in E-E_{0}} \arcsin \sqrt{\frac{\delta(a, f)}{2}}+k \\
& \leqslant \frac{\mu \pi}{\sqrt{2}}(n-k)+k \\
& \leqslant \begin{cases}n, & 0<\mu<\frac{\sqrt{2}}{\pi} \\
\frac{n \mu \pi}{\sqrt{2}}, & \frac{\sqrt{2}}{\pi} \leqslant \mu \leqslant \frac{1}{2} .\end{cases}
\end{aligned}
$$

This completes the proof of Theorem 1.

\section{Proof of Theorem 2}

We first assume that $\mu>0$ and define the sets of values

$$
\begin{aligned}
E & =\{a: \delta(a, f)>0\}, \\
E_{0} & =\{a: \delta(a, f) \geqslant 1-\cos \mu \pi\},
\end{aligned}
$$

where $E_{0}$ may be empty.

Since $\delta(a, f) \geqslant 1-\cos \mu \pi$ implies

$$
\frac{4}{\mu} \arcsin \sqrt{\frac{\delta(a, f)}{2}} \geqslant 2 \pi
$$

it follows from Lemma 1 that

$$
\sum_{a \in E-E_{0}} \frac{4}{\mu} \arcsin \sqrt{\frac{\delta(a, f)}{2}} \leqslant 2(n-p) \pi,
$$

where $p$ is the number of elements of $E_{0}$.

Notice that our assumption $\mu>0$ implies there are at least $n+1$ elements in $E$ (see [3]). We may assume that

$$
E-E_{0}=\left\{a_{1}, a_{2}, \ldots, a_{k} ; n-p+1 \leqslant k \leqslant \infty\right\}
$$

and (8) may be rewritten

$$
\sum_{i=1}^{k} \frac{2}{\pi \mu} \arcsin \sqrt{\frac{\delta\left(a_{i}, f\right)}{2}} \leqslant n-p .
$$


We now define quantities $d_{j}$ by the relations

$$
\delta\left(a_{j}, f\right)=2 d_{j} \sin ^{2} \frac{\pi \mu}{2}, \quad(j=1,2, \ldots, k)
$$

and confine our attention to

$$
0 \leqslant d_{j} \leqslant 1 \quad(j=1,2, \ldots, k) .
$$

Consider the function

$$
x=\varphi(s)=\left\{\frac{\sin (\pi \mu s / 2)}{\sin \pi \mu / 2}\right\}^{2} \quad(0 \leqslant s \leqslant 1)
$$

whose inverse is

$$
s=\varphi^{-1}(x)=\psi(x)=\frac{2}{\pi \mu} \arcsin \left\{x^{1 / 2} \sin \left(\frac{1}{2} \pi \mu\right)\right\}, \quad(0 \leqslant x \leqslant 1) .
$$

In view of (10) and (13), (9) takes the form

$$
\sum_{j=1}^{k} \psi\left(d_{j}\right) \leqslant n-p
$$

Notice that $\varphi(s)$ (defined by (12)) satisfies the conditions of Lemma 2, and therefore (11), (10), (14) and Lemma 2 show that

$$
\sum_{j=1}^{k} d_{j} \leqslant n-p, \quad \sum_{j=1}^{k} \delta\left(a_{j}, f\right) \leqslant(n-p)(1-\cos \pi \mu) .
$$

This gives

$$
\begin{aligned}
\sum_{a \in E} \delta(a, f) & =\sum_{a \in E-E_{0}} \delta(a, f)+\sum_{a \in E_{0}} \delta(a, f) \\
& \leqslant \sum_{j=1}^{k} \delta\left(a_{j}, f\right)+p \\
& \leqslant(n-p)(1-\cos \pi \mu)+p
\end{aligned}
$$

so that

$$
\sum_{a \in E} \delta(a, f) \leqslant n, \quad\left(0<\mu \leqslant \frac{1}{2}\right) .
$$

Next if $\mu=0$, it is known that $f(z)$ has at most $n$ deficient values [3], so that (15) is also true in this case. Theorem 2 is thus proved. 


\section{Sharpness of the Theorems}

Let $f(z)$ be defined by the following equation

$$
E(z) f^{n}-E(z)+1=0
$$

where $E(z)$ is an entire function of lower order $\mu(0 \leqslant \mu \leqslant 1 / 2)$. It is clear that $f(z)$ is an $n$-valued algebroid function of lower order $\mu$. Now let $a_{k}=\exp \{2 k \pi i / n\}$ $(k=1, \ldots, n)$. It follows that $N\left(r, a_{k}, f\right)$ is equal to zero and so $\delta\left(a_{k}, f\right)=1$ for $k=1,2, \ldots, n$.

The example mentioned above shows that the upper bound $n$ of the sums is sharp in our theorems if the algebroid function $f(z)$ has a small lower order.

REMARK. It is seen that the upper bound of the sum in Theorem 1 is much smaller than that in Theorem A, but we do not know if the result of Theorem 1 is best possible when $\mu>\sqrt{2} / \pi$. We also find in the theorems that equality is possible in Theorem 2 if and only if $f(z)$ has exactly $n$ deficient values $a_{i}(i=1,2, \ldots, n)$ such that $\delta\left(a_{i}, f\right)=1$, $i=1,2, \ldots, n$, when $0 \leqslant \mu \leqslant \sqrt{2} / \pi$.

\section{REFERENCES}

[1] A. Baernstein, 'Proof of Edrei's spread conjecture', Proc. London Math. Soc. 26 (1973), 418-434.

[2] A. Edrei, 'Solution of the deficiency problem for functions of small lower order', Proc. London Math. Soc. 26 (1973), 435-445.

[3] Y. Gu, 'The growth of algebroid functions with several deficient values', Contemp. Math. 25 (1983), 45-49.

[4] W.K. Hayman, Meromorphic functions (Oxford, 1964).

[5] R. Nevanlinna, Analytic functions (Springer-Verlag, Berlin, Heidelberg, New York, 1970).

[6] L-Z. Yang, 'Sums of deficiencies of algebroid functions', Bull. Austral. Math. Soc. 42 (1990), 191-200.

Department of Mathematics

Shandong University

Jinan

Shandong 250100

People's Republic of China 